\title{
Correction of the soft tissue problem using triamcinolone injection after rhinoplasty
}

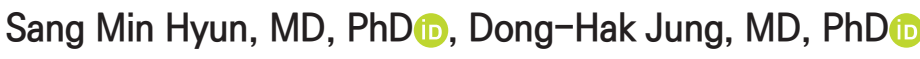 \\ Shimmian Rhinoplasty Clinic, Seoul, Rep. of Korea
}

\begin{abstract}
Background: Rhinoplasty aims to improve the function and the appearance of the nose by correcting and managing the cartilage framework. Despite correcting all the cartilage or bony deformities of the nose, the result is often unsatisfactory when the problem of skin soft tissue envelope occurs.

Objective: We have introduced a triamcinolone injection method for correcting the skin soft tissue envelopes after rhinoplasty. Methods: We retrospectively investigated 14 patients who were administered triamcinolone injection to manage skin soft tissue envelopes after rhinoplasty between September 2017 and March 2019. We began the injections at 4 weeks after the surgery and administered them 1 to 3 times at intervals of 6 weeks.

Results: Nine patients had re-growing bulbosity that enlarged the tip of the nose after surgery, 4 patients had asymmetry of the tip of the nose, and 1 had pollybeak deformity. Among these, 8 patients received one triamcinolone injection, 3 patients received it twice, and 3 patients received it up to 3 times. Twelve patients showed good results after the injection, while 2 patients had no improvement and underwent revision rhinoplasty 6 months after the first operation. None of the patients complained of other side effects.

Conclusion: Although rhinoplasty changes the function and the appearance mainly through structural deformation of the nose, there may be complaints due to unexpected deformation of the skin soft tissue envelope after surgery. Triamcinolone injection is thought to be a simple and effective way of improving the skin soft tissue envelope after surgery.
\end{abstract}

Keywords: bulbosity; pollybeak deformity; scarring; soft tissue envelope; triamcinolone

\section{Introduction}

Rhinoplasty is regarded as an effective and challenging cosmetic surgery that improves the nasal function. It mainly alters the structure of cartilage and bone, leading to esthetic and functional improvement. Many surgeons have tried and introduced various methods to improve the results and to reduce complications. Among different complications, the problem of soft tissue envelopes after surgery is a notorious one and a cause of anxiety for both patients and physicians. Re-growing bulbosity of the nasal tip and pollybeak deformity may result after rhinoplasty.
However, the complications may be unpredictable for even the most experienced surgeons [1]. The causes of these complications include inadequate management of cartilage, loss of tip support, and paradoxically excessive cartilage resection that results in subcutaneous scar tissue formation, often with thick nasal skin. If inadequate cartilage management is the cause, a simple revision such as trimming of the cartilage or reinforcement of tip support will solve the problem [2].

However, treatment may be more difficult if the cause is not a structural problem but a deformation of the soft tissue envelope after surgery. These deformations are often caused by filling of

Received December 2, 2019; Accepted December 14, 2019

Corresponding author: Sang Min Hyun

E-mail: hsm0313@gmail.com

This is an Open Access article distributed under the terms of the Creative Commons Attribution Non-Commercial License (http://creativecommons.org/licenses/by-nc/4.0), which permits unrestricted non-commercial use, distribution, and reproduction in any medium, provided the original work is properly cited.

Copyright $(2) 2019$ Korean Society of Korean Cosmetic Surgery and Medicine (KSKCS \& KCCS). 
scar tissue in dead spaces generated after cartilage reduction, especially in patients with thick skin. Results of revision surgery are also challenging to predict, since the revision may again cause dead space leading to scar tissue filling. Many surgeons have shown good results using triamcinolone acetonide injection to correct postoperative pollybeak deformity [3]. We present cases of triamcinolone injection treatment for the problem of postoperative skin soft tissue envelopes including pollybeak deformity.

\section{Materials and methods}

We retrospectively reviewed 14 patients who were administered triamcinolone injection to treat soft tissue envelope after rhinoplasty between September 2017 and March 2019. Patients (11 females and 3 males) had ages ranging from 19 to 36 years. Nine patients underwent primary rhinoplasty and 5 patients underwent revision rhinoplasty.

The inclusion criteria for the injections were enlarged or asymmetrical nasal tip at 4 weeks after the operation when compared with that at 2 weeks postoperatively and persistence of pollybeak deformity or bulbosity.

We injected 0.1 to $0.2 \mathrm{ml}$ of triamcinolone acetonide at a concentration of $10 \mathrm{mg} / \mathrm{ml}$ into the supratip area. The injections were administered in both the lateral supratip areas in case of re-growing bulbosity and in a single lateral supratip area in case of asymmetry. A single midline injection in the supratip area was administered for pollybeak deformity. The injections were administered in the subcutaneous tissue. Patients were assessed every 4 to 6 weeks after the injections and the need for subsequent injections was determined based on the effect of the previous injections. Each patient consented to its publication.

\section{Results}

Nine out of 14 patients had re-growing bulbosity that enlarged the tip of the nose after surgery, 4 patients had asymmetry of the tip of the nose, and 1 patient had pollybeak deformity. Among the patients with re-growing bulbosity, 5 patients showed acceptable results after the first triamcinolone acetonide injection (Fig. 1) and 2 patients showed improvement in bulbosity of the nose tip after the second injection. The remaining 2 patients showed little improvement after 3 injections and needed revision rhinoplasty to decrease the bulbosity of the nasal tip (Fig. 2).

Four out of the 14 patients had asymmetry of the supratip area at 1 month postoperatively, which was not observed at 2 weeks after the surgery. Among these, 3 patients showed symmetry of the supratip after the first injection and 1 patient showed acceptable symmetry after the second injection of triamcinolone acetonide (Fig. 3).

One patient had pollybeak deformity after rhinoplasty. We achieved an acceptable result with good supratip definition after 3 triamcinolone acetonide injections (Fig. 4).

There were no permanent complications from the triamcinolone injections in this study.

\section{Discussion}

Soft tissue problems after rhinoplasty may embarrass many surgeons and may reduce patient satisfaction. Revision surgery is often necessary in cases of re-growing bulbosity after bulbous nose correction, persistent pollybeak deformity, and asymmetry. Triamcinolone acetonide injection can improve the soft tissue problems after rhinoplasty with little side effects [4].

Triamcinolone injection is useful for the treatment of various diseases, especially for intradermal lesions such as keloids, hypertrophic scars, lichen planus, and psoriatic plaques. Triam-
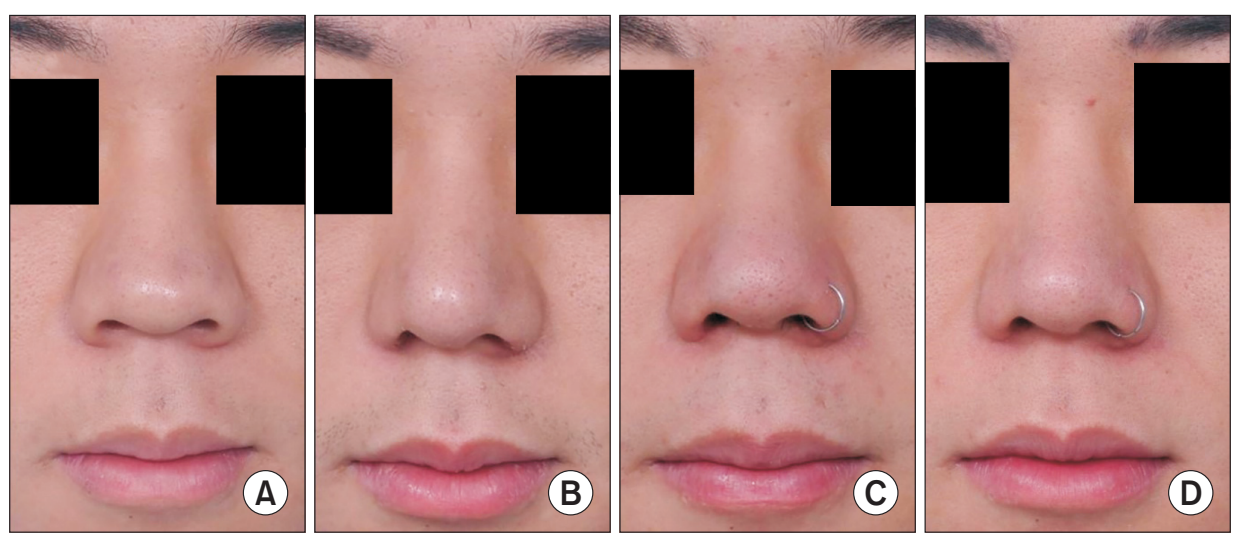

Fig. 1. After correction of bulbous nose, the nasal tip was enlarged 4 weeks after the operation, a finding not observed 2 weeks postoperatively. We achieved an acceptable result after the first injection of triamcinolone acetonide. (A) Preoperative photograph, (B) photograph taken 2 weeks postoperatively, (C) photograph taken 4 weeks postoperatively, and (D) photograph taken 10 weeks postoperatively and six weeks after the triamcinolone injection. 

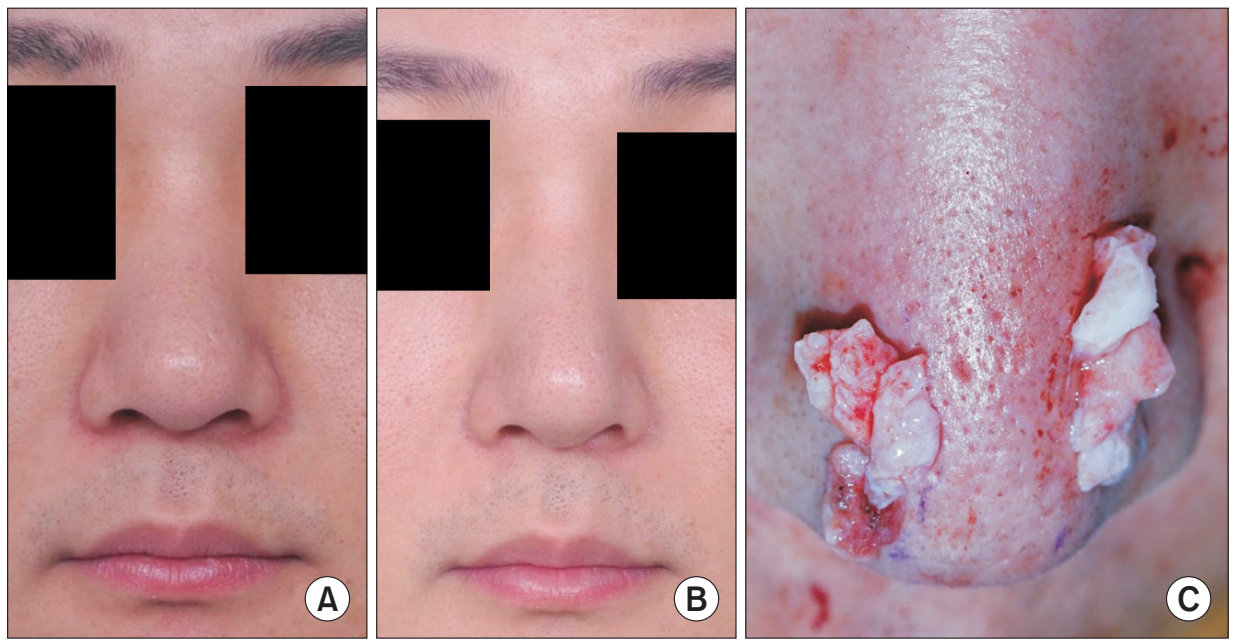

Fig. 2. A 28-year-old male patient showed little improvement in the re-growing bulbosity after the triamcinolone injection. We performed revision rhinoplasty including the debulking technique. (A) Preoperative photograph before revision rhinoplasty, (B) photograph taken 3 months postoperatively, and (C) fibrotic tissue removed through revision surgery.
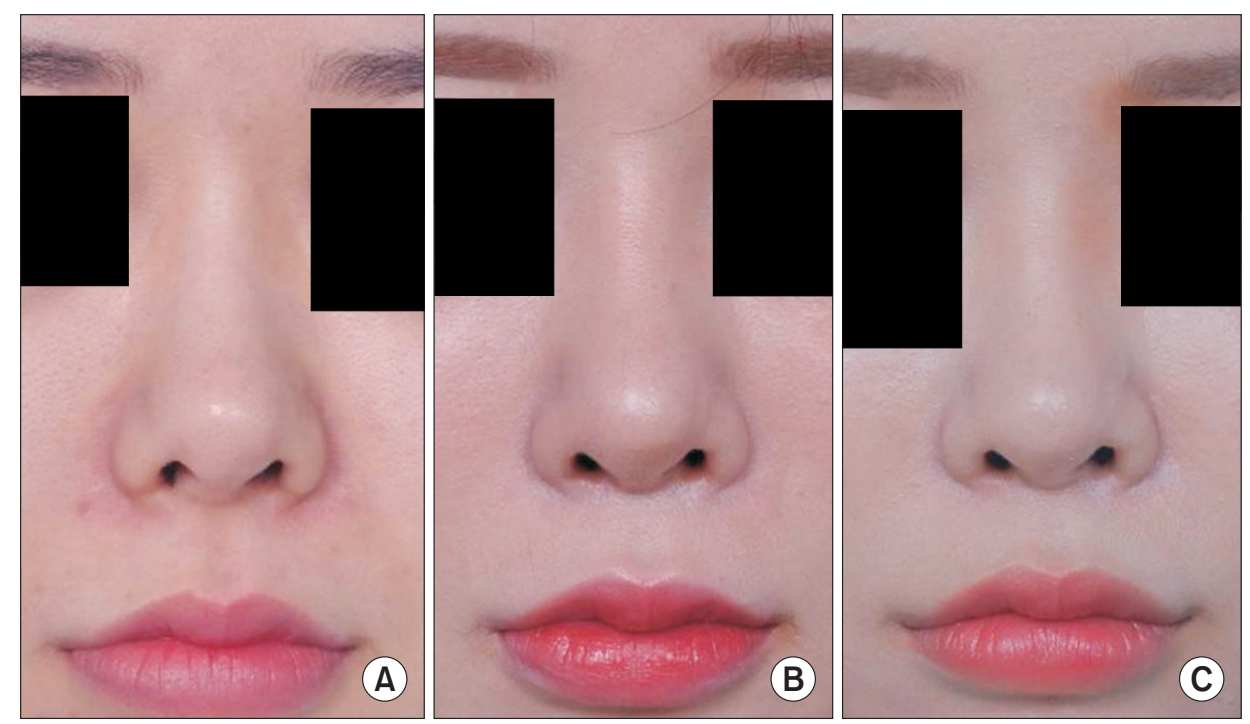

Fig. 3. We corrected the asymmetry of the supratip after rhinoplasty with triamcinolone injection on the left side of the supratip area. (A) Preoperative photograph, (B) photograph taken 3 months postoperatively, and (C) photograph taken 5 months postoperatively and 6 weeks after the triamcinolone injection.
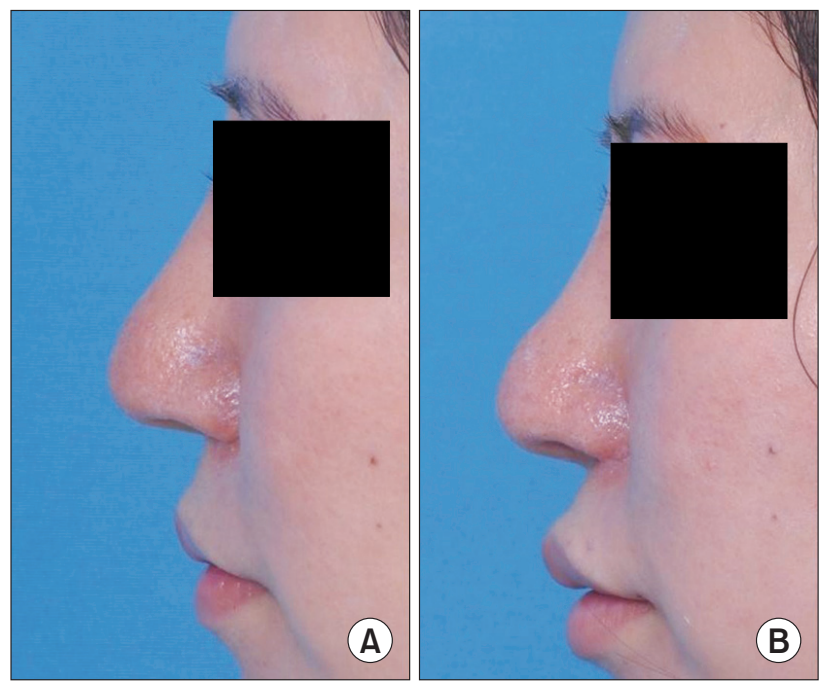

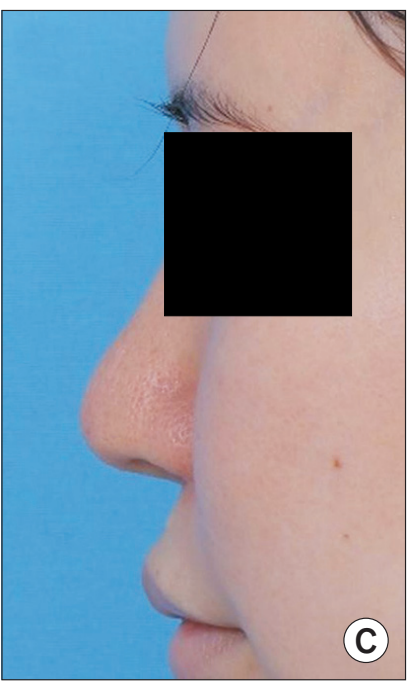

Fig. 4. Persistent pollybeak deformity after rhinoplasty was satisfactorily treated with 3 midline triamcinolone injections in the supratip area. (A) Preoperative photograph, (B) photograph taken 2 months postoperatively, and (C) photograph taken 7 months postoperatively and 5 months after the first triamcinolone injection. 
cinolone injection for keloids and hypertrophic scars was first described by Maguire [5] in 1965 and has been recommended as the first-line treatment to prevent and treat keloids and hypertrophic scars [6].

The exact mechanism by which triamcinolone acetonide reduces scar formation is not yet fully understood. It reduces fibroblast proliferation and inflammatory response, which in turn reduces synthesis of collagen and glycosaminoglycans and consequently reduces fibrosis. Moreover, since triamcinolone also increases collagen degradation, early treatment can result in suppression of proliferation as well as degradation [3].

In the present study, triamcinolone concentration was 10 $\mathrm{mg} / \mathrm{ml}$. Saedi et al. [7] compared the effects of triamcinolone on the supratip after rhinoplasty at different concentrations and reported that steroids were effective in reducing supratip edema. However, there were no significant differences in the effects according to different concentrations. Based on this study, we injected triamcinolone at a low concentration of $10 \mathrm{mg} / \mathrm{ml}$ and achieved good results without any side effects.

Potential complications of triamcinolone injection include subcutaneous atrophy, depigmentation, necrosis, and ulceration. Shafir et al. [8] described a case of blindness after steroid injection on the dorsum to treat soft tissue scarring. It was presumed to be caused by microembolus of injected suspension. To the best of our knowledge, no other cases of blindness were reported after injection on the dorsum and most of the cases of visual complications after triamcinolone injection were associated with the turbinate injection $[9,10]$. Nevertheless, to prevent blindness after triamcinolone injection, it is essential to aspirate while injecting to prevent injecting directly into a blood vessel. Since triamcinolone activity in the tissue lasts for 4 to 6 weeks, it is recommended that the interval between the injections should be 4 to 6 weeks to prevent the accumulation of side effects such as subcutaneous atrophy. Triamcinolone injection proved to be very effective in the present study. However, it requires a thorough understanding of the dosage, duration between the injections, and the technique to be strictly in the subcutaneous plane and never directly into the vessels to avoid potential risks and complications.

If the soft tissue deformity is not fully corrected even after 3 triamcinolone injections, revision surgery should be considered. During revision, scar tissue should be excised and compression tape and splint should be applied meticulously after the surgery. Compression tapes should be maintained for more than 3 weeks. From 2 weeks after the revision, triamcinolone injections are recommended 1 or 2 times to prevent recurrent subcutaneous scarring.
In conclusion, although rhinoplasty changes the function and the appearance mainly through structural deformation of the nose, there may be complaints due to unexpected deformation of the skin soft tissue envelope after the surgery. Since revision surgery is complicated and may cause other complications, triamcinolone injection should be considered as the first-line treatment for soft tissue deformity after rhinoplasty. It may reduce the need for revision and may increase patient satisfaction. If triamcinolone injection fails to treat the soft tissue deformity, revision rhinoplasty may be performed 6 months after the initial surgery.

\section{Conflicts of interest}

The authors have nothing to disclose.

\section{References}

1. Guyuron B, DeLuca L, Lash R. Supratip deformity: a closer look. Plast Reconstr Surg 2000;105:1140-51; discussion 1152-3.

2. Hussein WK, Foda HM. Pollybeak deformity in Middle Eastern rhinoplasty: prevention and treatment. Facial Plast Surg 2016;32:398-401.

3. Hanasono MM, Kridel RW, Pastorek NJ, Glasgold MJ, Koch RJ. Correction of the soft tissue pollybeak using triamcinolone injection. Arch Facial Plast Surg 2002;4:26-30; discussion 31.

4. Thomas WW, Bucky L, Friedman O. Injectables in the nose: facts and controversies. Facial Plast Surg Clin North Am 2016;24:379-89.

5. Maguire HC Jr. Treatment of keloids with triamcinolone acetonide injected intralesionally. JAMA 1965;192:325-6.

6. Ogawa R. The most current algorithms for the treatment and prevention of hypertrophic scars and keloids. Plast Reconstr Surg 2010;125:557-68.

7. Saedi B, Amali A, Arabpor M. Comparison of two concentrations of triamcinolone injection in the prevention of supratip edema after external rhinoplasty: a randomized trial. Am J Rhinol Allergy 2017;31:412-5.

8. Shafir R, Cohen M, Gur E. Blindness as a complication of subcutaneous nasal steroid injection. Plast Reconstr Surg 1999;104:1180-2; discussion 1183-4.

9. Martin PA, Church CA, Petti GH Jr, Hedayi R. Visual loss after intraturbinate steroid injection. Otolaryngol Head Neck Surg 2003;128:280-1.

10. Moss WJ, Kjos KB, Karnezis TT, Lebovits MJ. Intranasal steroid injections and blindness: our personal experience and a review of the past 60 years. Laryngoscope 2015;125:796-800. 